OPEN ACCESS

Edited by:

Uwe Knippschild,

University of Ulm, Germany

Reviewed by:

Timo Burster

Nazarbayev University, Kazakhstan

Jan Kroenke,

Ulm University Medical Center,

Germany

*Correspondence:

Ken Maes

ken.maes@vub.be

Specialty section:

This article was submitted to

Cell Death and Survival,

a section of the journal

Frontiers in Cell and Developmental

Biology

Received: 15 May 2019

Accepted: 17 July 2019

Published: 31 July 2019

Citation:

Maes K and Breckpot K (2019)

Commentary: Immunogenic Cell

Death and Immunotherapy of Multiple

Myeloma. Front. Cell Dev. Biol. 7:149.

doi: 10.3389/fcell.2019.00149

\section{Commentary: Immunogenic Cell Death and Immunotherapy of Multiple Myeloma}

\author{
Ken Maes ${ }^{1 *}$ and Karine Breckpot ${ }^{2}$ \\ 1 Department of Hematology and Immunology-Myeloma Center Brussels, Vrije Universiteit Brussel, Brussels, Belgium, \\ 2 Laboratory for Molecular and Cellular Therapy, Vrije Universiteit Brussel, Brussels, Belgium
}

Keywords: multiple myeloma, immunogenic cell death, immunotherapy, immunogenicity, tolerance

\section{A Commentary on}

Immunogenic Cell Death and Immunotherapy of Multiple Myeloma

by Serrano-Del Valle, A., Anel, A., Naval, J., and Marzo, I. (2019). Front. Cell Dev. Biol. 7:50. doi: $10.3389 /$ fcell.2019.00050

In April 2019, Serrano-del Valle and colleagues summarized the current applications of immunotherapy in the plasma cell malignancy multiple myeloma (MM) (Serrano-Del Valle et al., 2019). The authors moreover highlighted the connection of endoplasmic reticulum (ER) stress and the occurrence of immunogenic cell death (ICD). ICD is able to provoke potent adaptive immune responses and is based on the release of danger-associated molecular patterns (DAMPs) in a spatiotemporal manner (Garg et al., 2015). Interestingly, ER-stress pathways are important for DAMP exposure, including ecto-calreticulin, HMGB1, and ATP (Serrano-Del Valle et al., 2019). MM cells display enhanced ER-stress, hence making them dependent on ER-stress-related survival pathways. Compounds that target these survival pathways or that induce excessive amounts of ERstress are very effective in targeting MM cells. Therefore, it is tempting to speculate that ICD is an important mode of action of standard-of-care treatment.

To assess the occurrence of ICD in vivo, a vaccination assay can be performed in which dying tumor cells are used as a vaccine in mice (Kepp et al., 2014). In case bona fide ICD occurs, mice will be protected against a challenge with living tumor cells. We were the first to perform this vaccination assay using the syngeneic immunocompetent 5T33MM model (De Beck et al., 2018). The vaccine consisted of 5T33vt cells treated with bortezomib, melphalan, a DNA methyltransferase inhibitor (decitabine), a histone deacetylase inhibitor (quisinostat) or the combination of decitabine and quisinostat. We also used a well-known chemotherapeutic compound that induces ICD in solid cancers, i.e., mitoxantrone (Emeagi et al., 2012; Bezu et al., 2015). None of these treatments gave rise to a vaccine that provided $100 \%$ protection against MM outgrowth upon a subsequent challenge with living MM cells. This indicates that ICD did not occur in this model. When looking at the concept of ICD, it is important to note that bona fide ICD can only occur when tumor cells can expose the necessary DAMPs in response to treatment and when the recruited immune cells are not compromised in their function. However, when tumor cells cannot present all DAMPs, express tolerogenic molecules, or when immune cells cannot react properly on the DAMPs, ICD becomes inefficient resulting in tolerogenic cell death (TCD) (Garg et al., 2016). The occurrence of ICD in MM is influenced by the balance of tolerogenic and immunogenic molecules and the 
TABLE 1 | Current evidence of ICD hallmarks exposed by multiple myeloma cells.

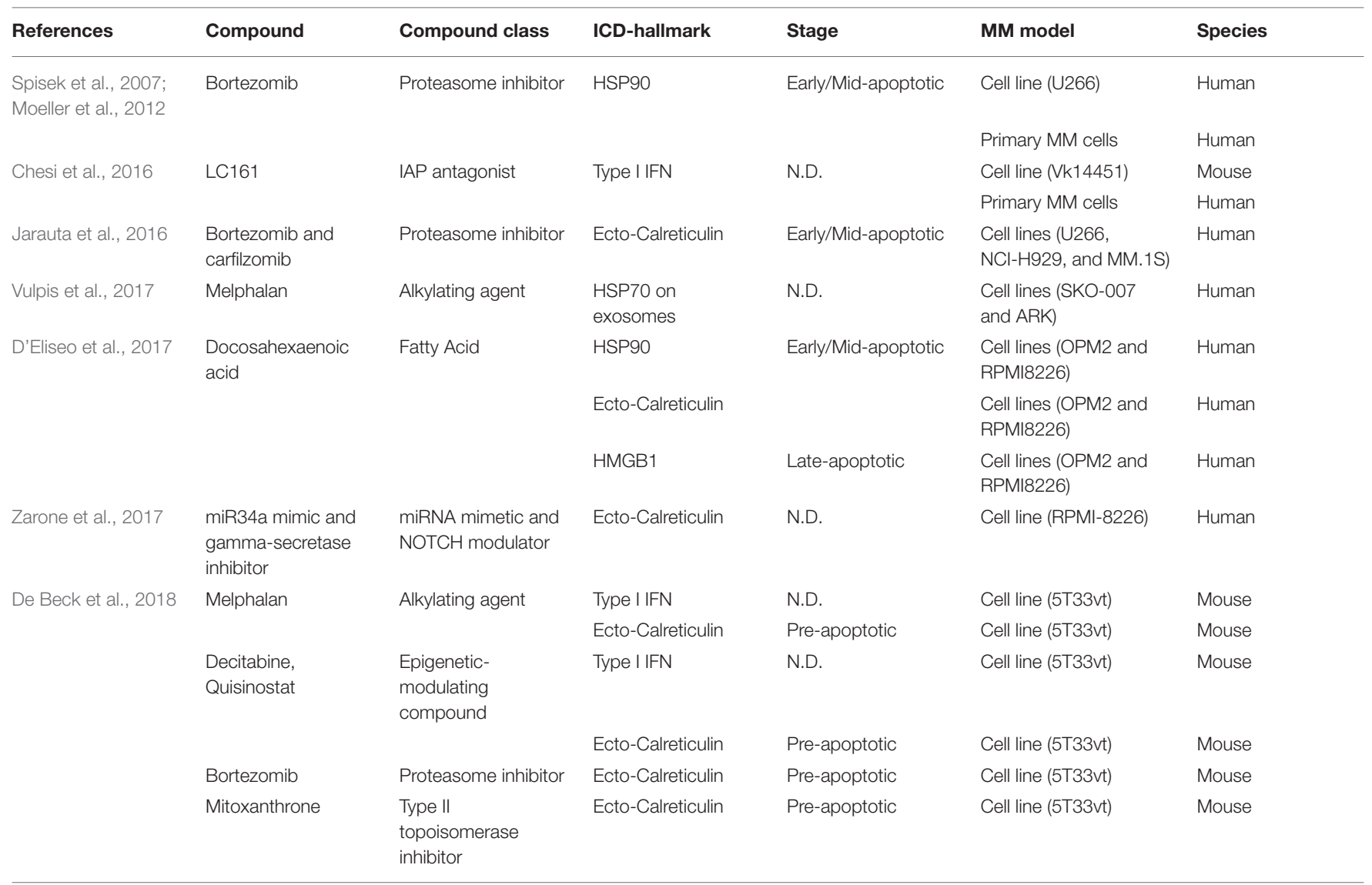

N.D, not determined.

associated receptors which are expressed by tumor and immune cells in the bone marrow (BM). In our study, we addressed DAMP exposure in 5T33vt cells. We showed that ectocalreticulin was present on a low amount of treated preapoptotic cells and that the "don't eat me signal" CD47 was highly expressed at basal and treatment conditions. None of the compounds increased the release of HMGB1. Decitabine, quisinostat, and melphalan induced a type I interferon response and induced signs of dendritic cell (DC) maturation upon co-culture of treated 5T33vt cells with BM monocyte-derived DCs. In vivo, epigenetic-modulating compounds increased ectocalreticulin and decreased CD47 expression in tumor cells, increased DC maturation, reduced CD11b-positive myeloid cells and transiently increased the amount of memory and naive $\mathrm{T}$ cells in the BM. Unfortunately, we could not unambiguously show that ICD occurred in the 5T33MM model as the vaccination assay did not provide $100 \%$ protection.

Other studies also focused on potential ICD effects as a modeof-action of standard-of-care compounds for MM patients and other compounds in pre-clinical studies (Table 1). Bortezomib induced an adaptive immune response in vitro with U266 and patient-derived MM cells in a HSP90 dependent manner (Spisek et al., 2007; Moeller et al., 2012). Carfilzomib treatment exposed calreticulin in 7-AAD-negative human myeloma cells
(Jarauta et al., 2016). Alkylating agents, including melphalan and cyclophosphamide, induced hallmarks of ICD including ecto-calreticulin and HMGB1 in thymoma, lymphoma, and colorectal cancer models (Schiavoni et al., 2011; Dudek-Perić et al., 2015; Lu et al., 2015). Other immune-related effects were also described, including depletion of regulatory $\mathrm{T}$ cells, induction of type I interferon, and increased effectiveness in conjunction with adoptive $\mathrm{T}$ cell transfer (Condomines et al., 2010; Sharabi and Haran-Ghera, 2011; Moschella et al., 2013). In MM, melphalan induced exosome release and Natural-Killer cell cytokine production in an HSP70-dependent manner (Vulpis et al., 2017). The fatty acid docosahexaenoic acid (DHA) increased ecto-calreticulin and HMGB1 release (D'Eliseo et al., 2017). Moreover, DHA-treated cells stimulated signs of maturation of ex vivo-generated DCs. The combination of a miR34 mimic and a gamma-secretase inhibitor induced exposure of calreticulin in MM cell lines (Zarone et al., 2017). At last, the IAP antagonist LC161 increased phagocytosis and induced a type I interferon response and long-lasting anti-MM immunity, independently of the presence of ecto-calreticulin (Chesi et al., 2016).

Despite these studies, progress on identifying hallmarks of ICD is limited and the ICD-inducing capacities of MM cells needs to be better defined. The question thus arises 
whether standard-of-care agents in MM evoke strong antiMM responses, especially because in MM, there is an inverse correlation between clinical outcome and mutational load, a parameter that at least in solid cancers is indicative for the success of many immunotherapies (Miller et al., 2017; Vitale et al., 2019). Furthermore, monocytes from MM patients show reduced efferocytosis (Liang et al., 2018). Monocyte-derived DCs from $\mathrm{MM}$ patients are ineffective in priming potent immune responses and plasmacytoid DCs have a pro-tumor phenotype by dampening $\mathrm{T}$-cell responses rather than stimulating them ( $\mathrm{Bi}$ et al., 2018; Shinde et al., 2018). Therefore, detailed examination of all molecular hallmarks of ICD and tolerogenic molecules on $\mathrm{MM}$ and immune cells in response to (combinations of) proteasome inhibitors, alkylating agents, immunomodulatory agents, dexamethasone, and monoclonal antibodies is warranted. This should be correlated to underlying stress responses (ERstress and autophagy) or genetic alterations in a larger number of human MM cell lines, immunocompetent murine models and

\section{REFERENCES}

Bezu, L., Gomes-de-Silva, L. C., Dewitte, H., Breckpot, K., Fucikova, J., Spisek, R., et al. (2015). Combinatorial strategies for the induction of immunogenic cell death. Front. Immunol. 6:187. doi: 10.3389/fimmu.2015.00187

Bi, E., Li, R., Bover, L. C., Li, H., Su, P., Ma, X., et al. (2018). E-cadherin expression on multiple myeloma cells activates tumor-promoting properties in plasmacytoid DCs. J. Clin. Invest. 128, 4821-4831. doi: 10.1172/JCI121421

Chesi, M., Mirza, N. N., Garbitt, V. M., Sharik, M. E., Dueck, A. C., Asmann, Y. W., et al. (2016). IAP antagonists induce anti-tumor immunity in multiple myeloma. Nat. Med. 22, 1411-1420. doi: 10.1038/nm.4229

Condomines, M., Veyrune, J. L., Larroque, M., Quittet, P., Latry, P., Lugagne, C., et al. (2010). Increased plasma-immune cytokines throughout the highdose melphalan-induced lymphodepletion in patients with multiple myeloma: a window for adoptive immunotherapy. J. Immunol. 184, 1079-1084. doi: 10.4049/jimmunol.0804159

De Beck, L., Melhaoui, S., De Veirman, K., Menu, E., De Bruyne, E., Vanderkerken, K., et al. (2018). Epigenetic treatment of multiple myeloma mediates tumor intrinsic and extrinsic immunomodulatory effects. Oncoimmunology. 7:e1484981. doi: 10.1080/2162402x.2018.1484981

D’Eliseo, D., Di Renzo, L., Santoni, A., and Velotti, F. (2017). Docosahexaenoic acid (DHA) promotes immunogenic apoptosis in human multiple myeloma cells, induces autophagy and inhibits STAT3 in both tumor and dendritic cells. Genes Cancer 8, 426-437. doi: 10.18632/genesandcancer.131

Dudek-Perić, A. M., Ferreira, G. B., Muchowicz, A., Wouters, J., Prada, N., Martin, S., et al. (2015). Antitumor immunity triggered by melphalan is potentiated by melanoma cell surface-associated calreticulin. Cancer Res. 75, 1603-1614. doi: 10.1158/0008-5472.CAN-14-2089

Emeagi, P. U., Van Lint, S., Goyvaerts, C., Maenhout, S., Cauwels, A., McNeish, I. A., et al. (2012). Proinflammatory characteristics of SMAC/DIABLOinduced cell death in antitumor therapy. Cancer Res. 72, 1342-1352. doi: 10.1158/0008-5472.CAN-11-2400

Garg, A. D., Galluzzi, L., Apetoh, L., Baert, T., Birge, R. B., Bravo-San Pedro, J. M., et al. (2015). Molecular and translational classifications of DAMPs in immunogenic cell death. Front. Immunol. 6:588. doi: $10.3389 /$ fimmu.2015.00588

Garg, A. D., Romano, E., Rufo, N., and Agostinis, P. (2016). Immunogenic versus tolerogenic phagocytosis during anticancer therapy: mechanisms and clinical translation. Cell Death Differ. 23, 938-951. doi: 10.1038/cdd.2016.5

Jarauta, V., Jaime, P., Gonzalo, O., de Miguel, D., Ramírez-Labrada, A., MartínezLostao, L., et al. (2016). Inhibition of autophagy with chloroquine potentiates carfilzomib-induced apoptosis in myeloma cells in vitro and in vivo. Cancer Lett. 382, 1-10. doi: 10.1016/j.canlet.2016.08.019
MM patient tumor and immune cells to obtain the broader picture of ICD and TCD. This is important as a misbalance between ICD and TCD toward TCD blunts therapy-induced immune priming and antitumor immune responses resulting in a worse outcome for patients.

\section{AUTHOR CONTRIBUTIONS}

All authors listed have made a substantial, direct and intellectual contribution to the work, and approved it for publication.

\section{FUNDING}

This work was funded by FWO-Vlaanderen, International Myeloma Foundation, Vrije Universiteit Brussel under the strategic research program scheme SRP48 and ERA-NET Transcan-2 JTC 2015 under research grant G0H7216N. KM is a postdoctoral fellow of FWO-Vlaanderen under grant 12E3819N.

Kepp, O., Senovilla, L., Vitale, I., Vacchelli, E., Adjemian, S., Agostinis, P., et al. (2014). Consensus guidelines for the detection of immunogenic cell death. Oncoimmunology. 3:e955691. doi: 10.4161/21624011.2014.955691

Liang, Y. Y., Schwarzinger, I., Simonitsch-Klupp, I., Agis, H., and Oehler, R. (2018). Impaired efferocytosis by monocytes in multiple myeloma. Oncol. Lett. 16, 409-416. doi: 10.3892/ol.2018.8620

Lu, X., Ding, Z. C., Cao, Y., Liu, C., Habtetsion, T., Yu, M., et al. (2015). Alkylating agent melphalan augments the efficacy of adoptive immunotherapy using tumor-specific CD4+ T cells. J. Immunol. 194, 2011-2021. doi: 10.4049/jimmunol.1401894

Miller, A., Asmann, Y., Cattaneo, L., Braggio, E., Keats, J., Auclair, D., et al. (2017). High somatic mutation and neoantigen burden are correlated with decreased progression-free survival in multiple myeloma. Blood Cancer J. 7:e612. doi: 10.1038/bcj.2017.94

Moeller, I., Spagnoli, G. C., Finke, J., Veelken, H., and Houet, L. (2012). Uptake routes of tumor-antigen MAGE-A3 by dendritic cells determine priming of naive T-cell subtypes. Cancer Immunol. Immunother. 61, 2079-2090. doi: 10.1007/s00262-012-1272-y

Moschella, F., Torelli, G. F., Valentini, M., Urbani, F., Buccione, C., Petrucci, M. T., et al. (2013). Cyclophosphamide induces a type I interferonassociated sterile inflammatory response signature in cancer patients' blood cells: implications for cancer chemoimmunotherapy. Clin. Cancer Res. 19, 4249-4261. doi: 10.1158/1078-0432.CCR-12-3666

Schiavoni, G., Sistigu, A., Valentini, M., Mattei, F., Sestili, P., Spadaro, F., et al. (2011). Cyclophosphamide synergizes with type I interferons through systemic dendritic cell reactivation and induction of immunogenic tumor apoptosis. Cancer Res. 71, 768-778. doi: 10.1158/0008-5472.CAN-10-2788

Serrano-Del Valle, A., Anel, A., Naval, J., and Marzo, I. (2019). Immunogenic cell death and immunotherapy of multiple myeloma. Front. Cell Dev. Biol. 7:50. doi: $10.3389 /$ fcell.2019.00050

Sharabi, A., and Haran-Ghera, N. (2011). Immune recovery after cyclophosphamide treatment in multiple myeloma: implication for maintenance immunotherapy. Bone Marrow Res. 2011:269519. doi: 10.1155/2011/269519

Shinde, P., Fernandes, S., Melinkeri, S., Kale, V., and Limaye, L. (2018). Compromised functionality of monocyte-derived dendritic cells in multiple myeloma patients may limit their use in cancer immunotherapy. Sci. Rep. 8:5705. doi: 10.1038/s41598-018-23943-w

Spisek, R., Charalambous, A., Mazumder, A., Vesole, D. H., Jagannath, S., and Dhodapkar, M. V. (2007). Bortezomib enhances dendritic cell (DC)-mediated induction of immunity to human myeloma via exposure of cell surface heat shock protein 90 on dying tumor cells: therapeutic implications. Blood 109, 4839-4845. doi: 10.1182/blood-2006-10-054221 
Vitale, I., Sistigu, A., Manic, G., Rudqvist, N. P., Trajanoski, Z., and Galluzzi, L. (2019). Mutational and antigenic landscape in tumor progression and cancer immunotherapy. Trends Cell Biol. 29, 396-416. doi: 10.1016/j.tcb.2019.01.003

Vulpis, E., Cecere, F., Molfetta, R., Soriani, A., Fionda, C., Peruzzi, G., et al. (2017). Genotoxic stress modulates the release of exosomes from multiple myeloma cells capable of activating NK cell cytokine production: role of HSP70/TLR2/NF-kB axis. Oncoimmunology 6:e1279372. doi: 10.1080/2162402X.2017.1279372

Zarone, M. R., Misso, G., Grimaldi, A., Zappavigna, S., Russo, M., Amler, E., et al. (2017). Evidence of novel miR-34a-based therapeutic approaches for multiple myeloma treatment. Sci. Rep. 7:17949. doi: 10.1038/s41598-017-18186-0
Conflict of Interest Statement: The authors declare that the research was conducted in the absence of any commercial or financial relationships that could be construed as a potential conflict of interest.

Copyright $\odot 2019$ Maes and Breckpot. This is an open-access article distributed under the terms of the Creative Commons Attribution License (CC BY). The use, distribution or reproduction in other forums is permitted, provided the original author(s) and the copyright owner(s) are credited and that the original publication in this journal is cited, in accordance with accepted academic practice. No use, distribution or reproduction is permitted which does not comply with these terms. 